\title{
Ultrasound Features for Determining the Risk of Malignancy in Unilocular-Solid Adnexal Masses in Premenopausal Women without Ascites and/or Carcinomatosis
}

\author{
${ }^{1}$ Leire Juez, ${ }^{2}$ Almudena Peces, ${ }^{3}$ Txanton Martinez-Astorquiza Corral, ${ }^{4}$ Rodrigo Orozco \\ ${ }^{5}$ Jesús Utrilla-Layna, ${ }^{6}$ María Caparros, ${ }^{7}$ Juan Luis Alcazar
}

\begin{abstract}
Purpose: To assess what clinical and ultrasound features could be most helpful for discriminating benign from malignant unilocular cysts with solid components in premenopausal women with no signs of ascites or carcinomatosis.
\end{abstract}

Materials and methods: Retrospective study comprising 91 women with preoperative diagnosis of unilocular-solid cyst that underwent surgical removal. The following variables were assessed: age, complaints at presentation, bilaterality, mean size of the lesion, number of papillary projections, size of solid component, surface of solid component (smooth or irregular), amount of color within solid component (no flow, minimal flow, moderate flow, abundant flow). Definitive histopathological diagnosis was used as gold standard. Multivariate logistic regression analysis was performed to identify which variables were independent predictors of malignancy and their odds ratios (OR).

Results: Malignant lesions had significantly larger tumor size, more number of solid components, larger size of solid component, more frequency of irregular surface in the solid component and more frequent moderate or abundant vascularization within the solid component. Logistic regression analysis identified amount of color (OR: 48.7), solid component's surface (OR: 29.1) and mean size of the lesion (OR: 1.58) as independent predictors for malignancy.

Conclusion: Tumor size, irregular surface and the presence of moderate or abundant color score within the solid component are the features more frequently associated to malignancy in unilocular-solid adnexal cysts in premenopausal women.

Keywords: Malignancy, Premenopause, Ultrasound, Unilocular cyst.

How to cite this article: Juez L, Peces A, Corral TMA, Orozco R, Utrilla-Layna J, Caparros M, Alcazar JL. Ultrasound

\footnotetext{
1,2,4-6 Resident, ${ }^{3}$ Specialist (Ob/Gyn), ${ }^{7}$ Full Professor

${ }^{1,5-7}$ Department of Obstetrics and Gynecology, Clínica Universidad de Navarra, School of Medicine, University of Navarra Pamplona, Spain

2,4 Department of Obstetrics and Gynecology, University Hospital Virgen de la Arrixaca, Murcia, Spain

${ }^{3}$ Department of Obstetrics and Gynecology, University Hospital Universitario Cruces, Bilbao, Spain
}

Corresponding Author: Juan Luis Alcazar, Department of Obstetrics and Gynecology, Clínica Universidad de Navarra Avenida Pío XII 36, 3110 Pamplona, Spain, Phone: +34-948296234, Fax: +34-948296500, e-mail: jlalcazar@unav.es
Features for Determining the Risk of Malignancy in UnilocularSolid Adnexal Masses in Premenopausal Women without Ascites and/or Carcinomatosis. Donald School J Ultrasound Obstet Gynecol 2015;9(2):112-117.

Source of support: Nil

Conflict of interest: None

\section{INTRODUCTION}

Transvaginal ultrasound has been shown as an excellent diagnostic tool for discriminating benign from malignant adnexal masses when performed by expert examiners. ${ }^{1-3}$ Most ovarian cancers appear as solid or multilocularsolid masses, many times with signs of intra-abdominal spread, such as ascites or frank carcinomatosis. ${ }^{4-6}$ On the contrary, unilocular cysts are rarely malignant lesions. ${ }^{7}$

The finding of solid tissue within an unilocular cyst rises the suspicion of a possible malignancy. However, many benign masses exhibit this ultrasound appearance. ${ }^{8-10}$ For this reason, an unilocular cyst with solid components represents an actual diagnostic challenge. In fact, some studies conclude that this type of adnexal mass is the most difficult to categorize when no other signs of malignancy like ascites or carcinomatosis are present. ${ }^{11,12}$

When managing surgically ovarian lesions of uncertain nature, such as unilocular-solid cysts, the surgeon must choose between adnexectomy and cystectomy. This decision is important for fertility sparing surgery but bearing in mind that albeit ovarian cancer is not common in premenopausal women, but it does happen. ${ }^{13}$

Therefore, attempts for improving our diagnostic performance in this type of lesions in premenopausal women are advisable.

The aim of this study was to assess what clinical and ultrasound features could be most helpful for discriminating benign from malignant unilocular cysts with solid components in premenopausal women.

\section{MATERIALS AND METHODS}

This is a retrospective study performed at a single university tertiary care center. Institutional review board waived patient consent and gave approval. 
A search in our database for identifying women who underwent surgery for an adnexal masses at our center between January 1995 and December 2014 was performed.

Eligible patients were premenopausal women preoperatively diagnosed as having an adnexal mass categorized as 'unilocular-solid cyst' and evaluated by transvaginal sonography (TVS). We defined as 'unilocular-solid cyst' any cyst with a predominantly cystic component and solid excrescences with height of at least $3 \mathrm{~mm}$ but no upper limit of size protruding into the cyst cavity ${ }^{14}$ (Fig. 1).

Clinical, ultrasound and histopathological records were reviewed by two of the authors (JLA, LJ). Stored video clips and/or 3D volumes from the masses were also retrieved for review.

We excluded women with incomplete data from clinical records, ultrasound report and histopathological report. We also excluded women if ultrasound report described the presence of ascites or carcinomatosis, were pregnant at diagnosis or no hard-print copies, video clips and/or 3D volumes were available for review. Finally, we excluded those cases that after reviewing hard-print copies, video clips and/or 3D volumes the mass did not fit the definition of 'unilocular-solid cyst'.

Ultrasound stored video clips and/or 3D volumes were reviewed by three sonologists who were blinded to histopathological results (AP, TMA, RO). All of them had with more than 2 years' experience in gynecological ultrasound and were trained in ultrasound assessment of adnexal masses by one of the authors (JLA).

The following variables were retrospectively assessed:

- Clinical records: age, complaints at presentation.

- Histopathological report: Definitive histological diagnosis. This data were used as gold standard. Malignancies were staged according to FIGO classification. ${ }^{15}$ Borderline ovarian tumors (BOTs) were considered as malignant for analytical purposes.

- Ultrasound features:

- Bilaterality.

- Mean size of the lesion as determined by measuring tumor diameters in the three orthogonal planes.

- Number of solid excrescences or papillary projections (Fig. 2).

- Maximum size of solid excrescences or papillary projections as measured by two perpendicular planes (Fig. 3). In case of two or more solid excrescences or papillary projections we considered the largest one for analytical purposes.

- Surface of solid excrescence or papillary projections, stated as smooth or irregular (Figs 4 and 5). In case of two or more solid excrescences or papillary projections with at least one exhibiting smooth and at least other with irregular surface

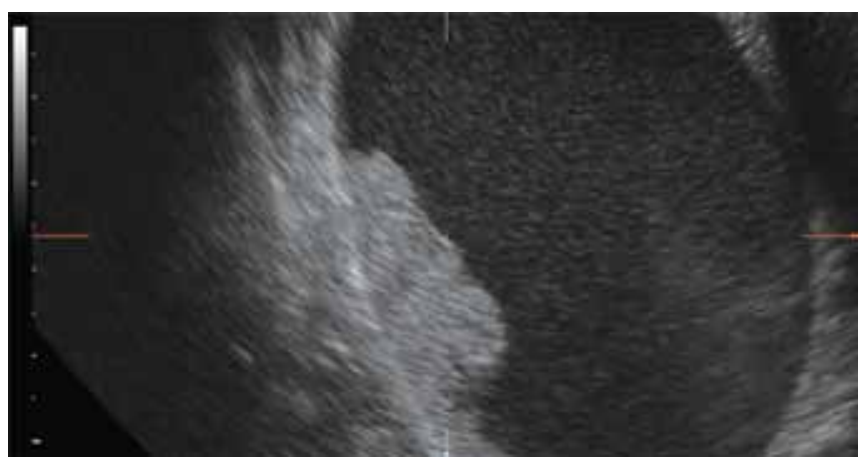

Fig. 1: Transvaginal ultrasound from a unilocular-solid cyst. A solid area is observed arising from internal cyst wall and protruding into the cyst's cavity

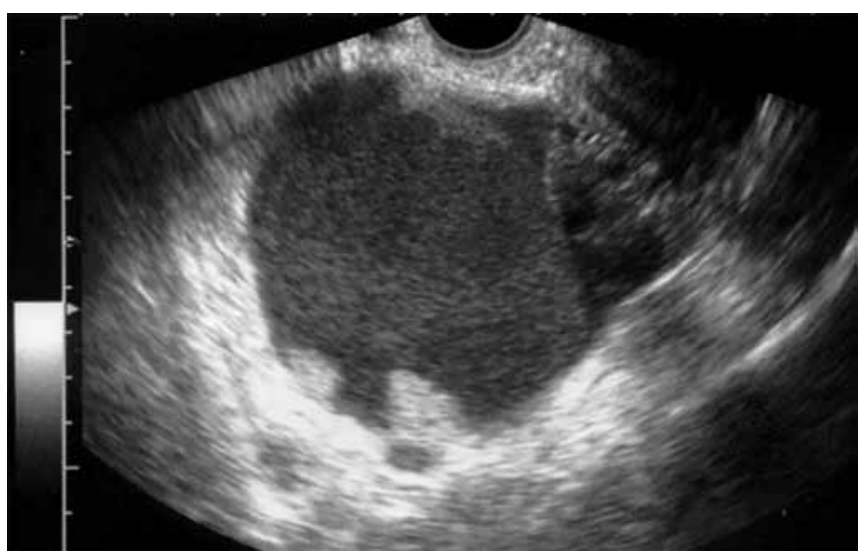

Fig. 2: Transvaginal ultrasound from a unilocular-solid cyst with two solid papillary projections of different size

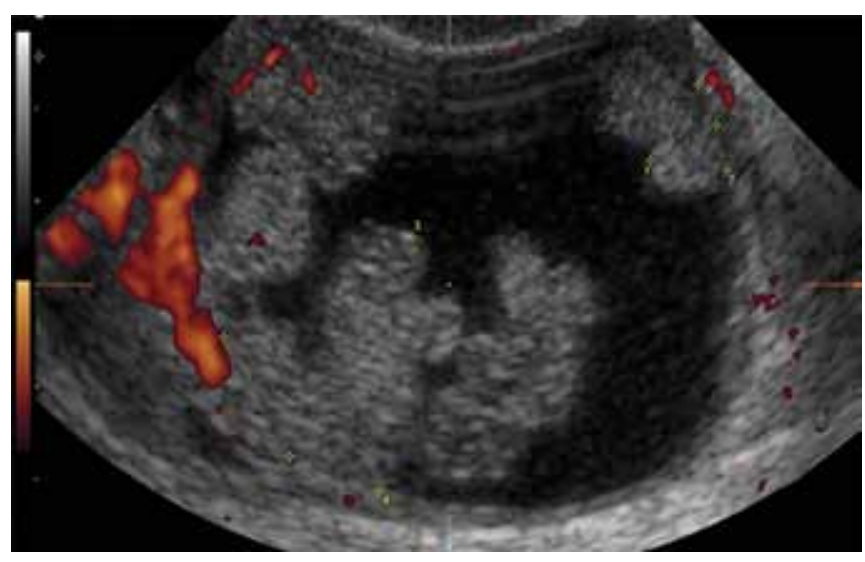

Fig. 3: Measurement of papillary projections in an unilocularsolid cyst. Height and perpendicularly the width

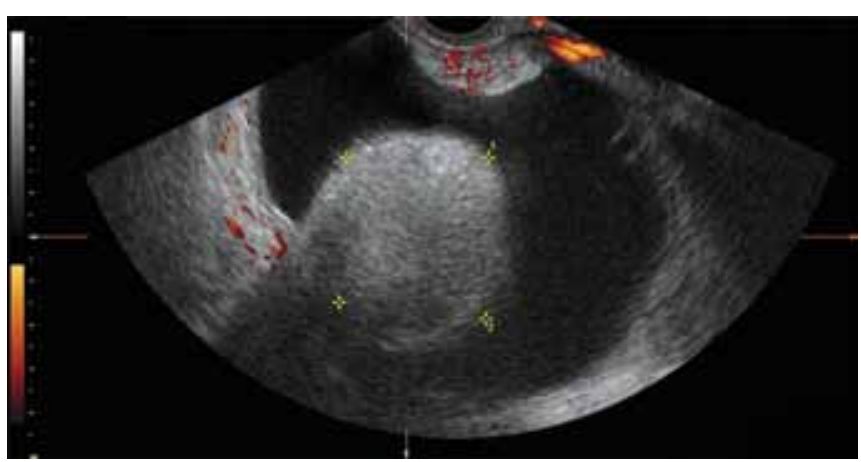

Fig. 4: Transvaginal ultrasound from a unilocular-solid cyst depicting a large solid area with smooth surface 


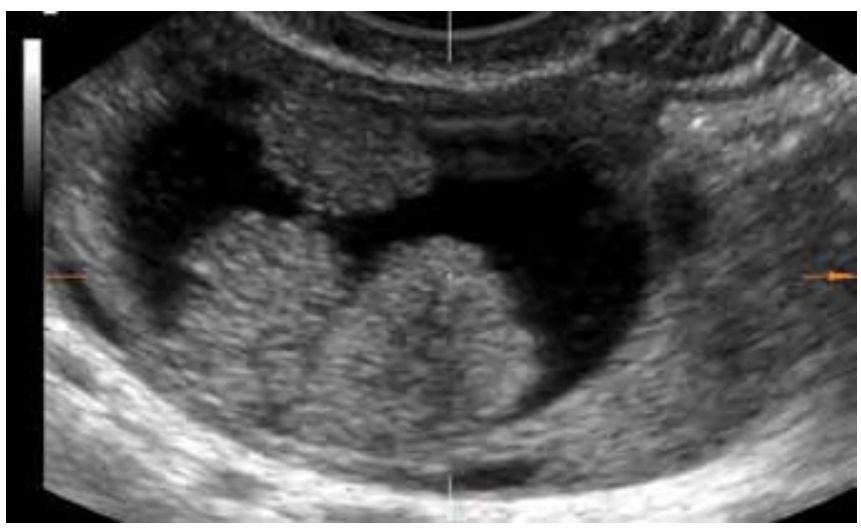

Fig. 5: Unilocular-solid cyst exhibiting three papillary projections with irregular surface

we consider only the irregular one for analytical purposes.

- Amount of color score within papillary projections as defined by IOTA ${ }^{14}$ : Color score (1) no flow, color score (2) minimal flow, color score (3) moderate flow and color score (4) abundant flow (Figs 6 and 7).

All ultrasound examinations had been performed or supervised by one examiner (JLA) according to a predefined scanning protocol described elsewhere. ${ }^{16,17}$

Kolmogorov-Smirnov test was used to assess normal distribution of continuous variables. Data are presented as mean with standard deviation (SD) or median with interquartile range (IQR).

Categorical variables were expressed as absolute number and percentage.

Univariate analysis was first performed to determine whether statistical differences between benign and malignant lesions exist for each individual variable. Continuous variables were compared using one-way ANOVA or Mann-Whitney U tests depending whether variables distributed normally or not. Categorical variables were compared using Chi-squared test. Those variables with statistical differences were then entered in a forward stepwise multivariate logistic regression analysis to identify which variables were independent predictors of malignancy and to determine their odds ratios with 95\% confidence intervals (CIs).

A p-value less than 0.05 was considered as statistically significant for all analyses. Statistical package for social sciences (SPSS) version 22.0 was used for all statistical analyses.

\section{RESULTS}

From January 1995 to December 2014, 2135 women underwent surgery for an adnexal mass at our institution. After database search, we found out 91 women eligible for the study. Thirty-eight women were excluded (incomplete

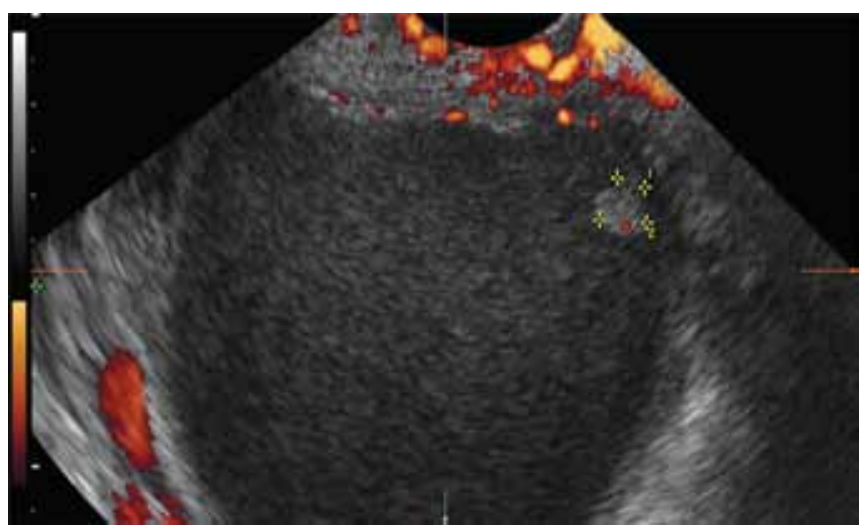

Fig. 6: Transvaginal ultrasound from a unilocular-solid cyst showing a small papillary projection with minimal color signals (color score 2)

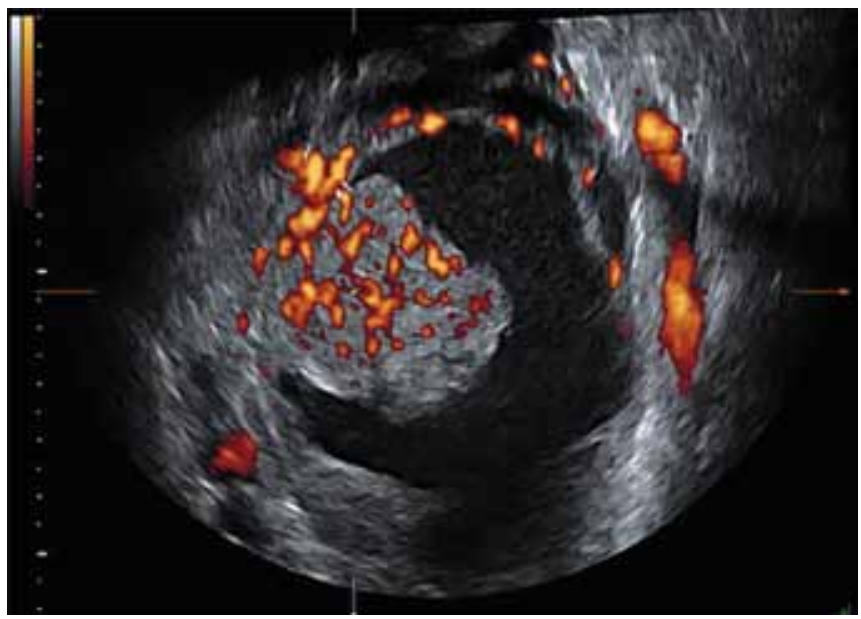

Fig. 7: Transvaginal ultrasound from a unilocular-solid cyst showing a large papillary projection with abundant color signals (color score 4)

data: $\mathrm{n}=17$; presence of ascites or carcinomatosis: $\mathrm{n}=6$; no video clips and/or 3D volumes available for review, $\mathrm{n}=9$; the mass did not fit definition of unilocular-solid cyst after hard-print copies, video clips and/or 3D volumes review, $\mathrm{n}=4$; and were pregnant at diagnosis, $\mathrm{n}=2$ ).

Ultimately, 53 women were included in this study. Patients' mean age was 38 years old (SD: 8.6), ranging from 17 to 52 years.

Complaints at presentation were as follows: asymptomatic, $\mathrm{n}=38$ (72.0\%); pelvic/abdominal pain, $\mathrm{n}=10$ $(18.9 \%)$; menstrual disorder, $\mathrm{n}=3(5.4 \%)$ and abdominal swelling, $\mathrm{n}=2$ (3.7\%).

Five women had bilateral masses $(9.4 \%)$. However, none of them had a bilateral unilocular-solid mass.

Mean diameter of the lesions was $67.5 \mathrm{~mm}$ (SD: 27.0), ranging from $17.0 \mathrm{~mm}$ to $139.5 \mathrm{~mm}$. Median number of solid excrescences or papillary projections was 2 (IQR: 2), ranging from 1 to 8 . Mean largest diameter of the solid component was $25.3 \mathrm{~mm}$ (SD: 21.1), ranging from 3.5 to $92.5 \mathrm{~mm}$. 
Irregular surface of the solid component was present in 30 cases (56.6\%) and it was smooth in 23 cases ( $43.3 \%$ ).

Color score was absent in 14 cases (26.4\%), minimal in 24 cases $(45.3 \%)$, moderate in 8 cases $(15.1 \%)$ and abundant in 7 cases (13.2\%).

At histopathology analysis, 15 tumors $(28.3 \%)$ were malignant and $38(71.7 \%)$ were benign (Table 1$)$. Tumor stage of malignant tumors were as follows: stage Ia, $\mathrm{n}=$ $6(40.0 \%)$; stage Ib: $\mathrm{n}=1(6.7 \%)$; stage Ic, $\mathrm{n}=3(20 \%)$ and stage IIIc, $\mathrm{n}=5$ (33.3\%).

Univariate analysis is shown in Table 2. We found that malignant lesions had significantly larger tumor size, more number of solid components, larger size of solid component, more frequency of irregular surface in the solid component and more frequent moderate or abundant vascularization within the solid component.

Table 1: Histopathological diagnosis of the lesions

\begin{tabular}{lll}
\hline Histology & $N$ & Percent \\
\hline Cystadenofibroma & 11 & 20.8 \\
Endometrioma & 7 & 13.2 \\
Teratoma & 6 & 11.3 \\
Serous cystadenoma & 5 & 9.2 \\
Mucinous cystadenoma & 5 & 9.4 \\
Hemorrhagic cyst & 3 & 5.7 \\
Peritoneal cyst & 1 & 1.9 \\
BOT lesion & 4 & 7.5 \\
Invasive carcinoma & 11 & 20.8 \\
\hline BOT: Borderline ovarian tumor & &
\end{tabular}

BOT: Borderline ovarian tumor

Table 2: Results of univariate statistical analysis

\begin{tabular}{|c|c|c|c|}
\hline Variable & $\begin{array}{l}\text { Benign } \\
(n=38)\end{array}$ & $\begin{array}{l}\text { Malignant } \\
(n=15)\end{array}$ & $p$-value \\
\hline $\mathrm{Age}^{*}$ & $37.8(9.1)$ & $38.7(7.4)$ & 0.735 \\
\hline Complaints $^{\dagger}$ & & & 0.315 \\
\hline Asymptomatic & $28(73.7)$ & $10(66.7)$ & \\
\hline Pelvic pain & $6(15.8)$ & $3(20.0)$ & \\
\hline Menstrual disorder & $3(7.9)$ & $0(0)$ & \\
\hline $\begin{array}{l}\text { Abdominal } \\
\text { swelling }\end{array}$ & $1(2.6)$ & $2(13.3)$ & \\
\hline Bilaterality $^{\dagger}$ & $2(5.3)$ & $3(20.0)$ & 0.131 \\
\hline Tumor size $(\mathrm{cm})^{\star}$ & $5.9(2.1)$ & $8.9(3.1)$ & 0.001 \\
\hline $\begin{array}{l}\text { Number solid } \\
\text { components }^{\ddagger}\end{array}$ & $1.0(0.0)$ & $2.0(2.0)$ & 0.001 \\
\hline $\begin{array}{l}\text { Solid component } \\
\text { size }(\mathrm{mm})^{\ddagger}\end{array}$ & $14.3(16.5)$ & $30.0(38.4)$ & 0.002 \\
\hline $\begin{array}{l}\text { Solid component } \\
\text { surface }{ }^{\dagger}\end{array}$ & & & 0.001 \\
\hline Irregular & $16(42.1)$ & $14(93.3)$ & \\
\hline Smooth & $22(57.9)$ & $1(6.7)$ & \\
\hline Color score & & & 0.001 \\
\hline Absent/scanty & $34(89.5)$ & $4(26.7)$ & \\
\hline $\begin{array}{l}\text { Moderate/ } \\
\text { abundant }\end{array}$ & $4(10.5)$ & $11(73.3)$ & \\
\hline
\end{tabular}

*Expressed as mean, standard deviation in parentheses; †Expressed as absolute number, percentage in parentheses;

‡Expressed as median, interquartile range in parentheses
Multivariate logistic regression analysis showed that only color score (odd ratio: 48.7, 95\% CIs: 3.9-602.3; $\mathrm{p}=$ 0.002), irregular surface (odd ratio: 29.1, 95\% CIs: 1.4-601.9; $\mathrm{p}=0.029$ ) and mean size of the lesion (odd ratio: $1.6,95 \%$ CIs: 1.1-2.5; $\mathrm{p}=0.039)$ were independent predictors for malignancy. The presence of one moderate/abundant color score or irregular surface of the solid component identified $100 \%$ of malignant lesions with a false-positive rate of $34 \%$.

\section{DISCUSSION}

In the literature, there are many different prediction models for preoperative assessment of ovarian lesions. Some were using morphological, the others Doppler characterization, some were using both. Some even used 3D ultrasound..$^{18}$ However, it seems that subjective assessment by an expert using pattern recognition appears remains the best method of distinguishing between benign and malignant tumors. ${ }^{1}$

Some studies even used computational systems based on machine-learning techniques, such as artificial neural networks and support vector machines..$^{19}$ However, they did not confer additional discriminating power. Moreover, there is no evidence that any other imaging modality (such as CT, PET or PET CT) will perform any better than does ultrasound. ${ }^{20}$

In this study, we have assessed what clinical and ultrasound parameters could discriminate better between benign and malignant unilocular-solid adnexal cysts in premenopausal women without other obvious suspicious signs such as ascites and/or carcinomatosis. We have found that irregular surface of the solid component and the amount of color signals within the solid component are the best predictors for malignancy.

The main strength of this study is that we provide some new information that could be used by sonographers and sonologists. In fact, there is a significant paucity of data regarding this type of lesions in the literature. To the best of our knowledge, there is only a previous study that specifically addressed the ultrasound predictors for malignancy in unilocular-solid cysts in premenopausal women.

Fagotti et al evaluated 51 premenopausal women with unilocular-solid adnexal cysts to determine the diagnostic performance of different ultrasound parameters for discriminating benign from malignant lesions. ${ }^{21}$ They found that a largest solid component $>$ $14 \mathrm{~mm}$ and the presence of blood flow within the solid component, as detected by color Doppler, provided a $100 \%$ sensitivity and $80 \%$ specificity for distinguishing benign and BOT lesions from invasive carcinoma. 
There are some similarities between Fagotti's and ours study. Both are retrospective analysis, both included a similar number of cases ( 51 and 53, respectively) and both had a similar prevalence of BOT and invasive carcinomas (30 and $28.3 \%$, respectively).

However, there are some important differences. Probably, the main one is how both studies grouped the cases. Fagotti et al analyzed benign and BOT lesions vs invasive carcinomas. Whereas we analyzed benign tumors vs BOT and invasive carcinomas.

We consider this is an important difference from the clinical point of view. In premenopausal women benign adnexal lesions should be treated laparoscopically by the most conservative approach (cystectomy) whenever possible. ${ }^{22}$ On the other hand, albeit fertility-sparing surgery may be considered, invasive cancer should be treated more radically by adnexectomy and complete surgical staging. ${ }^{23}$ Borderline ovarian tumors may be treated by cystectomy, the when using this approach the risk of recurrence is significantly higher and the patient must be informed..$^{24}$ For this reason, we think that for diagnostic work-up and patient counseling BOT tumors should be considered as malignant. Even more when distinguishing preoperatively between invasive cancer and BOT is actually a difficult task. ${ }^{25}$

Like Fagotti et $\mathrm{al}^{21}$ we found that the number of solid components or papillary projections and the largest diameter of the solid component were statistically significant larger in malignant tumors. However, in our study this statistical difference is lost in the multivariate logistic regression analysis.

Regarding color score we found that most BOT/Invasive cancers had moderate or abundant vascularization within the solid component. However, the presence of minimal and moderate color signals were observed in many benign lesions. Fagotti et al however, reported that just the presence/absence of color signals is a good discriminator. It could be argued that this controversial result could be explained by the subjective nature of color score assessment. However, at least two studies have shown that IOTA color score is reproducible among different examiners. ${ }^{26,27}$

Additionally, Fagotti et al did not find differences in surface of the solid component (irregular versus smooth). However, Hassen et $\mathrm{al}^{28}$ and Valentin et $\mathrm{al}^{29}$ also found that irregular surface of the solid component was predictive of malignancy (considering BOT and invasive carcinoma as malignant tumors in their respective statistical analysis). Notwithstanding, these two latter studies included pre- and postmenopausal women and did not reported specific data for premenopausal women.

In our opinion, these contradictory findings might be explained by the different criteria used for selecting and grouping cases.
Our study, obviously, had limitations. The main one is its retrospective design. This fact causes many exclusions and, therefore, a selection bias.

Second, the series is too small to allow us to draw definitive conclusions. For this reason, we did not estimate diagnostic performance and did not attempted to develop a predictive model.

According to this, we could conclude that irregular surface of the solid component and the presence of moderate or abundant color score within the solid component are those features more frequently associated to BOT or invasive carcinoma in unilocular-solid adnexal cysts in premenopausal women without ascites and/or carcinomatosis.

\section{REFERENCES}

1. Valentin L. Pattern recognition of pelvic masses by gray-scale ultrasound imaging: the contribution of Doppler ultrasound. Ultrasound Obstet Gynecol 1999;14(5):338-347.

2. Sokalska A, Timmerman D, Testa AC, et al. Diagnostic accuracy of transvaginal ultrasound examination for assigning a specific diagnosis to adnexal masses. Ultrasound Obstet Gynecol 2009;34(4):462-470.

3. Alcázar JL, Guerriero S, Mínguez JÁ, et al. Adding cancer antigen 125 screening to gray scale sonography for predicting specific diagnosis of benign adnexal masses in premenopausal women: is it worthwhile? J Ultrasound Med 2011;30(10):1381-1386

4. Granberg S, Norström A, Wikland M. Tumors in the lower pelvis as imaged by vaginal sonography. Gynecol Oncol 1990;37(2):224-229.

5. Timmerman D, Schwärzler P, Collins WP, et al. Subjective assessment of adnexal masses with the use of ultrasonography: an analysis of interobserver variability and experience. Ultrasound Obstet Gynecol 1999;13(1):11-16.

6. Alcázar JL, Guerriero S, Laparte C, Ajossa S, Jurado M. Contribution of power Doppler blood flow mapping to gray-scale ultrasound for predicting malignancy of adnexal masses in symptomatic and asymptomatic women. Eur J Obstet Gynecol Reprod Biol 2011;155(1):99-105.

7. Valentin L, Ameye L, Franchi D, et al. Risk of malignancy in unilocular cysts: a study of 1148 adnexal masses classified as unilocular cysts at transvaginal ultrasound and review of the literature. Ultrasound Obstet Gynecol 2013;41(1):80-89.

8. AlcázarJL, Errasti T, Mínguez JA, Galán MJ, García-Manero M, Ceamanos C. Sonographic features of ovarian cystadenofibromas: spectrum of findings. J Ultrasound Med 2001;20(8): 915-919.

9. Guerriero S, Alcazar JL, Ajossa S, et al. Transvaginal color Doppler imaging in the detection of ovarian cancer in a large study population. Int J Gynecol Cancer 2010;20(5):781-786.

10. Brown DL, Dudiak KM, Laing FC. Adnexal masses: US characterization and reporting. Radiol 2010;254(2):342-354.

11. Valentin L, Ameye L, Jurkovic D, et al. Which extrauterine pelvic masses are difficult to correctly classify as benign or malignant on the basis of ultrasound findings and is there a way of making a correct diagnosis? Ultrasound Obstet Gynecol 2006;27(4):438-444. 
12. Valentin L, Ameye L, Savelli L, et al. Adnexal masses difficult to classify as benign or malignant using subjective assessment of gray-scale and Doppler ultrasound findings: logistic regression models do not help. Ultrasound Obstet Gynecol 2011;38(4):456-465.

13. Heintz AP, Odicino F, Maisonneuve P, et al. Carcinoma of the ovary. FIGO 26th annual report on the results of treatment in gynecological cancer. Int J Gynaecol Obstet 2006;95(Suppl 1):S161-192.

14. Timmerman D, Valentin L, Bourne TH, Collins WP, Verrelst $\mathrm{H}$, Vergote I. International ovarian tumor analysis group: terms, definitions and measurements to describe the sonographic features of adnexal tumors-a consensus opinion from the international ovarian tumor analysis group. Ultrasound Obstet Gynecol 2000;16(5):500-505.

15. Mutch DG, Prat J. FIGO staging for ovarian, fallopian tube and peritoneal cancer. Gynecol Oncol 2014;133(3):401-404.

16. Alcázar JL, Errasti T, Laparte C, Jurado M, López-García G. Assessment of a new logistic model in the preoperative evaluation of adnexal masses. J Ultrasound Med 2001;20(8):841-848.

17. Alcázar JL, Mercé LT, García Manero M. Three-dimensional power Doppler vascular sampling: a new method for predicting ovarian cancer in vascularized complex adnexal masses. J Ultrasound Med 2005;24(5):689-696.

18. Kurjak A, Kupesic S, Breyer B, Sparac V, Jukic S. The assessment of ovarian tumor angiogenesis: what does threedimensional power Doppler add? Ultrasound Obstet Gynecol 1998;12(2):136-146.

19. Van Calster B, Timmerman D, Lu C, et al. Preoperative diagnosis of ovarian tumors using Bayesian kernel-based methods. Ultrasound Obstet Gynecol 2007;29(5):496-504.

20. American College of Obstetricians and Gynecologists. ACOG Practice Bulletin. Management of adnexal masses. Obstet Gynecol 2007;110(1):201-214
21. Fagotti A, Ludovisi M, De Blasis I, et al. The sonographic prediction of invasive carcinoma in unilocular-solid ovarian cysts in premenopausal patients: a pilot study. Hum Reprod 2012;27(9):2676-2683.

22. Nezhat C, Cho J, King LP, Hajhosseini B, Nezhat F. Laparoscopic management of adnexal masses. Obstet Gynecol Clin North Am 2011;38(4):663-676.

23. Zapardiel I, Diestro MD, Aletti G. Conservative treatment of early stage ovarian cancer: oncological and fertility outcomes. Eur J Surg Oncol 2014;40(4):387-393.

24. Uzan C, Muller E, Kane A, et al. Prognostic factors for recurrence after conservative treatment in a series of 119 patients with stage I serous borderline tumors of the ovary. Ann Oncol 2014;25(1):166-171.

25. Exacoustos C, Romanini ME, Rinaldo D, et al. Preoperative sonographic features of borderline ovarian tumors. Ultrasound Obstet Gynecol 2005;25(1):50-59.

26. Zannoni L, Savelli L, Jokubkiene L, et al. Intra- and interobserver reproducibility of assessment of Doppler ultrasound findings in adnexal masses. Ultrasound Obstet Gynecol 2013;42(1):93-101.

27. Pineda L, Salcedo E, Vilhena C, Juez L, Alcázar JL. Interobserver agreement in assigning IOTA color score to adnexal masses using three-dimensional volumes or digital videoclips: potential implications for training. Ultrasound Obstet Gynecol 2014;44(3):361-364.

28. Hassen K, Ghossain MA, Rousset P, et al. Characterization of papillary projections in benign versus borderline and malignant ovarian masses on conventional and color Doppler ultrasound. Am J Roentgenol 2011;196(6):1444-1449.

29. Valentin L, Ameye L, Savelli L, et al. Unilocular adnexal cysts with papillary projections but no other solid components: is there a diagnostic method that can classify them reliably as benign or malignant before surgery? Ultrasound Obstet Gynecol 2013;41(5):570-581. 